\title{
Surface Conductance near the Order-Disorder Phase Transition on Si(100)
}

\author{
Kwonjae Yoo $^{1}$ and H.H. Weitering ${ }^{2}$ \\ ${ }^{1}$ Department of Physics and Astronomy, The University of Tennessee, Knoxville, Tennessee 37996 \\ and Solid State Division, Oak Ridge National Laboratory, Oak Ridge, Tennessee 37831 \\ ${ }^{2}$ Department of Applied Physics and DIMES, Delft University of Technology, Lorentzweg 1, 2628 CJ Delft, The Netherlands \\ (Received 9 June 2000; published 21 June 2001)
}

\begin{abstract}
The surface conductance of the $\mathrm{Si}(100)-(2 \times 1)$ surface was measured as a function of temperature on a fully depleted $\mathrm{Si}(100) / \mathrm{SiO}_{2} / \mathrm{Si}$ substrate. The surface-state conductance is surprisingly large and reveals a clear signature of the $c(4 \times 2) \rightarrow 2 \times 1$ order-disorder phase transition of buckled Si dimers on $\mathrm{Si}(100)$. Surface scattering increases with decreasing $c(4 \times 2)$ order on the surface.
\end{abstract}

DOI: $10.1103 /$ PhysRevLett.87.026802

The transport properties of solids - their capacity to conduct heat and electricity - have always been a core topic of condensed matter physics [1]. Important discoveries in condensed matter and materials physics are most often associated with novel transport phenomena, frequently encountered in materials of considerable chemical and structural complexity or materials of reduced dimensionality. Ordered surfaces and ultrathin films are a special class of low-dimensional materials that are quantum confined in one dimension but extended periodically in the other two dimensions. Surface science has provided the know-how and means to fabricate and characterize atomically clean surfaces and to manipulate thin film growth and thus presents wonderful opportunities to explore the fundamentals of electronic transport in reduced dimensionality. However, the present understanding of surface electronic properties is based on electron spectroscopies and firstprinciples electronic structure calculations. None of these relates directly to the macroscopic materials properties such as the temperature-dependent electrical conductivity, which so far seemed impossible to measure.

In this Letter, we present temperature-dependent fourpoint probe conductivity measurements of the $\mathrm{Si}(100)$ $(2 \times 1)$ surface. This reconstructed surface consists of a two-dimensional array of buckled Si dimers atop the bulktruncated surface (Fig. 1a) [2]. At the lowest measurement temperature $(120 \mathrm{~K})$, buckled Si dimers are locked into an "antiferromagnetic" $c(4 \times 2)$ array. As the temperature is raised, the long-ranged $c(4 \times 2)$ order diminishes gradually due to thermally activated flip-flop motions of the $\mathrm{Si}$ dimers [2] and/or rapid propagation of phase-shifting defects along the dimer rows [3]. The order-disorder transition is complete near $200 \mathrm{~K}$. This two-dimensional (2D) electron system is characterized by a $\sim 0.5 \mathrm{eV}$ gap separating filled and empty surface-state bands, a total electron density of $6.78 \times 10^{14} \mathrm{~cm}^{-2}$, and an effective mass of $\sim 0.4 m_{e}$ [2]. The Fermi level is pinned by defectinduced gap states associated with "type- $C$ " point defects (Fig. 1b) [4].

Random adsorbates on a thin metal film scatter conduction electrons and increase the electrical resistivity of the film [5,6]. Therefore, in order to measure the
PACS numbers: 73.25. $+\mathrm{i}$, 68.35.Bs, 68.35.Rh

transport properties intrinsic to a surface or ultrathin film, experiments must be carried out in ultrahigh vacuum (UHV). UHV transport measurements on thin metal films have been reported [7-11], but the temperature dependence of the electrical conductivity has not been studied in the ultimate limit of a reconstructed surface layer. We have utilized bulk $\mathrm{Si}$ and ultrathin $\mathrm{Si} / \mathrm{SiO}_{2} / \mathrm{Si}$ or silicon-on-insulator (SOI) substrates to analyze the relative importance of the various conduction channels near the $\mathrm{Si}(100)$ surface as a function of temperature. The surface-state conductivity appears surprisingly large and exhibits a metallic temperature dependence between 120 and $300 \mathrm{~K}$. The data furthermore show a clear signature of the $c(4 \times 2) \rightarrow 2 \times 1$ orderdisorder transition of buckled Si dimers on $\mathrm{Si}(100)$ [2].

Experiments were carried out in an UHV. We employed $n$-type $\mathrm{Si}(100)$ bulk wafer $(14 \Omega \mathrm{cm})$ and $p$-type

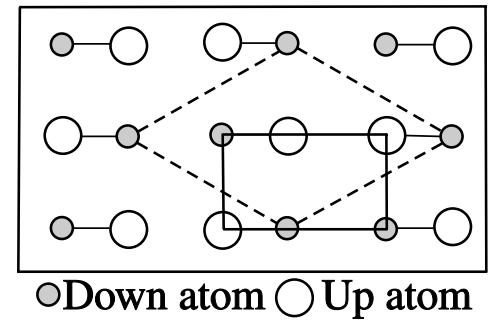

(a)

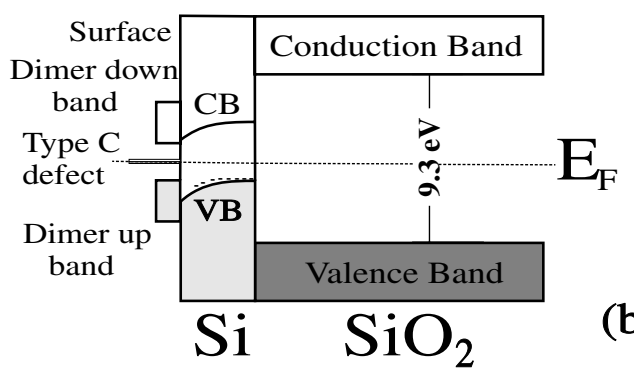

FIG. 1. (a) Top view of the $2 \times 1$ dimer array on $\mathrm{Si}(100)$. At RT, dimer atoms oscillate up-down, resulting in a time-averaged $2 \times 1$ structure (solid line). Dashed lines indicate the primitive unit cell of the $c(4 \times 2)$ structure. (b) Schematic band diagram of the silicon-on-insulator structure including the surface states and the type- $C$ defect state (not to scale). 
(100) SOI material $(19 \Omega \mathrm{cm})$. The SOI material has a top layer thickness of $900 \AA$ (which is well below the nominal depletion layer width of $\approx 7000 \AA$ ) and was prepared and characterized according to the procedures in Ref. [12]. Ohmic contact pads $\left(2 \times 2 \mathrm{~mm}^{2}\right)$ were defined by a $10^{15} \mathrm{~cm}^{-2}$ phosphorous or boron ion implant on the four corners of a $10 \times 10 \mathrm{~mm}^{2}$ sample. The sheet resistance was measured according to the van der Pauw method using a dc current source. A well-ordered $\operatorname{Si}(100)-(2 \times 1)$ surface reconstruction was obtained by repeated flashing to $1300 \mathrm{~K}$ in UHV as evidenced with low energy electron diffraction (LEED).

The surface conductance, $\Delta \sigma(T)$, in units of $\Omega^{-1} / \square$, is an excess quantity defined as follows:

$$
G(T)=\sigma_{b}(T) \times d+\Delta \sigma(T),
$$

where $G(T)$ is the square conductance of the sample, $d$ is the thickness of the sample, and $\sigma_{b}(T)$ is the conductivity of the bulk. $\Delta \sigma(T)$ includes electronic conduction through the surface states and through the space-charge layer beneath the surface and vanishes for an ideally truncated surface with no surface states. The space-charge layer beneath the $\operatorname{Si}(100)-(2 \times 1)$ surface is a depletion layer due to Fermi level pinning by type- $C$ surface defects at $\sim 0.4 \mathrm{eV}$ above the bulk valence band maximum (Fig. 1b) $[4,12]$.

To obtain $\Delta \sigma(T)$ for $\operatorname{Si}(100)-(2 \times 1)$, we first measured the square conductance $G_{2 \times 1}(T)$ of the $n$-type bulk $\mathrm{Si}(100)-(2 \times 1)$ sample in UHV from $T \approx 120$ to $300 \mathrm{~K}$. Next, we saturated the clean $\operatorname{Si}(100)-(2 \times 1)$ surface with $\mathrm{O}_{2}$ at RT. This procedure destroys the surface reconstruction (as evidenced by a $1 \times 1$ LEED pattern), eliminates the surface states [13], and creates a nearly flat-band surface according to band bending measurements with $\mathrm{Si}$ $2 p$ core level spectroscopy [14]. The conductance of the oxidized sample, $G_{\mathrm{O}_{2}}(T)$, was also measured from 120 to $300 \mathrm{~K}$ and we obtained $\Delta \sigma(T)=G_{2 \times 1}(T)-G_{\mathrm{O}_{2}}(T)$. This difference measurement is prone to large errors if the contact resistances are not exactly reproducible after cooling and heating cycles. The use of ion-implanted contacts is therefore pivotal. To avoid possible hysteresis, we recorded data only during the heating of the sample. In reality, $G_{\mathrm{O}_{2}}(T)$ is slightly less than $\sigma_{b}(T) \times d$ due to adsorbate-induced surface scattering. We show, however, that this effect is negligible for $\mathrm{Si}$.

Figure 2 shows the conductance of the $(2 \times 1)$ reconstructed bulk $\mathrm{Si}$ sample as a function of exposure to $\mathrm{O}_{2}$ at RT. It shows that a clean reconstructed sample is less resistive than a sample passivated with $\mathrm{O}_{2}$, in

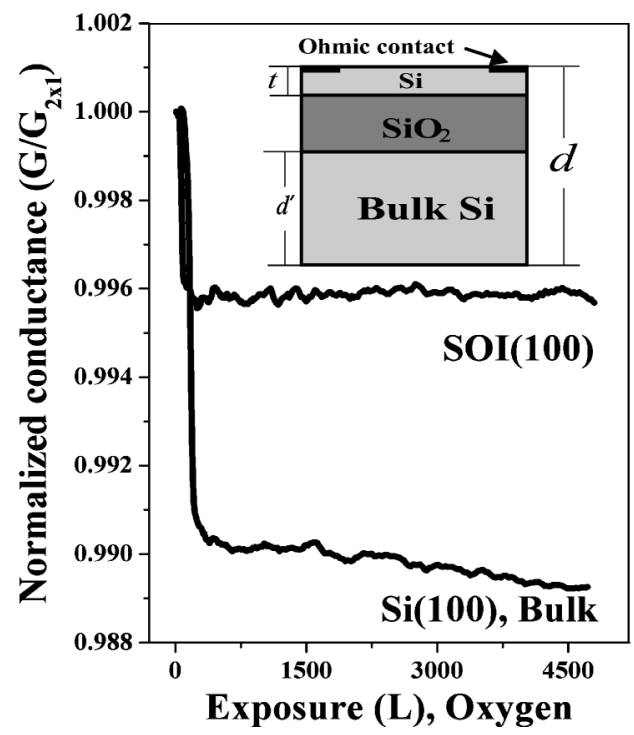

FIG. 2. Conductance of $n$-type bulk $\operatorname{Si}(100)-(2 \times 1)$ and $p$-type $\mathrm{SOI}(100)$ during exposure to $\mathrm{O}_{2}$ at RT. Inset: Side view of the SOI sample with ion-implanted contacts.

agreement with other RT chemisorption studies $[15,16]$. The same behavior has been observed for $p$-type samples [15] and has been explained in terms of space-charge layer conductance [16] or diffuse scattering at the $\mathrm{O}_{2}$ passivated surface [15]. As we show below, none of these mechanisms accounts for the experimental observations. Instead, the data of Fig. 2 are fully consistent with the presence of a surface-state conduction channel on $\operatorname{Si}(100)-(2 \times 1)$ that is gradually destroyed by the adsorption of $\mathrm{O}_{2}$.

In order to separate the surface-state and space-charge layer contributions to the total surface conductance, $\Delta \sigma(T)$, we calculate the space-charge layer contribution, $\Delta \sigma_{s c}(T)$, using Ref. [17]:

$$
\Delta \sigma_{s c}=e\left(\mu_{n, s} \Delta N+\mu_{p, s} \Delta P\right),
$$

where $\Delta N=\int_{o}^{\infty}\left[n(z)-n_{b}\right] d z$ and $\Delta P=\int_{o}^{\infty}[p(z)-$ $\left.p_{b}\right] d z$ are the excess densities of electrons $(n)$ and holes $(p)$, respectively, near the surface. Subscripts $s$ and $b$ refer to the surface $(z=0)$ and bulk $(z=\infty)$, respectively; $\mu_{n, s}$ and $\mu_{p, s}$ are the surface mobilities. By solving Poisson's equation in the abrupt depletion approximation using Boltzmann statistics and parabolic bands and by introducing the new variables $v(z) \equiv\left[E_{i b}-E_{i}(z)\right] / k_{B} T$ and $u(z) \equiv\left[E_{F}-E_{i}(z)\right] / k_{B} T$, Kingston and Neustadter derived [18]

$$
\Delta N=n_{b} L_{D} \int_{v_{s}}^{0}\left(e^{v}-1\right) / \mp F\left(u_{b}, v\right) d v,
$$

$$
F\left(u_{b}, v\right)=\sqrt{2} \sqrt{\left[\cosh \left(u_{b}+v\right) / \cosh u_{b}-v \tanh u_{b}-1\right]}
$$

(with a similar equation for $\Delta P$ ), $E_{i}(z)$ is the intrinsic Fermi level, $v(z)$ is the band bending $\left[v_{s} \approx 0.34 \mathrm{eV}\right.$ for $\operatorname{Si}(100)-(2 \times 1)$ at $\mathrm{RT}], L_{D}$ is the extrinsic Debye length, and $k_{B}$ is Boltzmann's constant. The surface mobility of
$\operatorname{Si}(100)-(2 \times 1)$ was calculated for the majority carriers using the approximate solution of the Boltzmann transport equation for depletion layers $[17,19]$ : 


$$
\mu_{s}=\mu_{b}\left\{1-\frac{(1-r) \lambda_{b}}{L_{D} \sqrt{2}} \frac{1-e^{-\left|v_{s}\right|}}{\sqrt{\left(e^{-\left|v_{s}\right|}+\left|v_{s}\right|-1\right)}}\right\},
$$

where $\mu_{b}$ is the mobility of the majority carrier in the bulk and $\lambda_{b} \approx\left(\frac{\mu_{n, b}}{e}\right) \sqrt{\left(3 m^{*} k_{B} T\right)}$ is the bulk mean free path ( $m^{*}$ is the electron effective mass). The phenomenological Fuchs parameter $r$ represents the fraction of specular scattering events [20]. Its temperature dependence depends on the microscopic nature of the surface scattering events, i.e., scattering from surface phonons, ionized impurities, and/or surface roughness. We have calculated the temperature-dependent $\Delta \sigma_{s c}(T)$ for the $\operatorname{Si}(100)-(2 \times 1)$ surface from Eqs. (2) - (5), taking into account the temperature dependences of the parameters $\lambda_{b}, L_{D}, v_{s}, u_{b}$, $\mu_{b}$, and $n_{b}$ for specular $(r=1)$ and diffuse $(r=0)$ surface scattering. Minority carrier contributions have been ignored. The theoretical $\Delta \sigma_{s c}(T)$ is shown in Fig. 3, together with the experimental data $\Delta \sigma(T)$. Irrespective of the precise surface scattering mechanism, $\Delta \sigma_{s c}(T)$ is opposite in sign to the experimental $\Delta \sigma(T)$. This discrepancy increases at lower temperature. Evidently, the surface-state conduction $\Delta \sigma_{s s}(T)$ contributes to the total sheet conductance. It is obtained by subtracting $\Delta \sigma_{s c}$ from the experimental surface conductance, $\Delta \sigma(T)$ (see Fig. 3).

In a previous RT study [15], it was proposed instead that the discrepancy between $\Delta \sigma_{s c}$ and $\Delta \sigma$ is caused by a strongly reduced carrier mobility beneath the $\mathrm{O}_{2}$ passivated surface due to roughening of the surface during oxidation; i.e., the $\mathrm{O}_{2}$-passivated surface may not be representative of the ideal flat-band surface. A simple argument shows that this effect is small and does not reverse

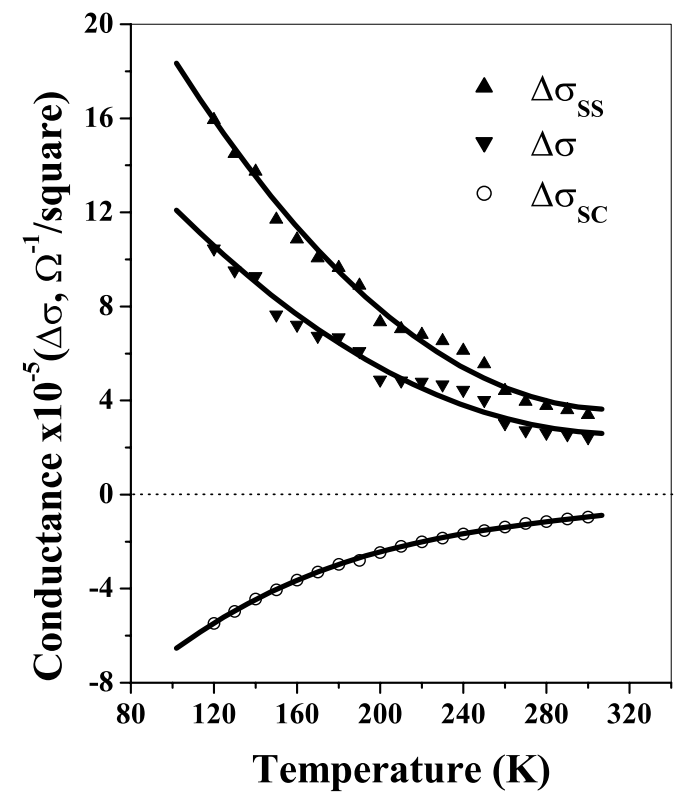

FIG. 3. Temperature dependence of the excess surface conductance according to the space-charge layer calculation, $\Delta \sigma_{s c}(T)$, and according to experiment, $\Delta \sigma$, for the $n$-type bulk $\mathrm{Si}(100)$ sample. The surface-state conductance $\Delta \sigma_{s s}(T)=\Delta \sigma(T)-$ $\Delta \sigma_{s c}(T)$. the sign of $\Delta \sigma(T)$. In the abrupt depletion approximation, extrinsic carriers originating from the $2 \times 1$ depletion layer (width $\sim L_{D}$ ) are trapped in the surface states so that $G_{2 \times 1} \approx \sigma_{b}\left(d-L_{D}\right)$. In the limit of completely diffuse scattering $(r=0)$ at the flat-band $\mathrm{O}_{2} / \mathrm{Si}(100)$ surface, one obtains $G_{\mathrm{O}_{2}} \approx \sigma_{b}\left(d-\lambda_{b}\right)$. Since $\lambda_{b} \ll L_{D}$ for $\mathrm{Si}$ at RT and below, $\Delta \sigma(T)=\left\{G_{2 \times 1}(T)-G_{\mathrm{O}_{2}}(T)\right\}<0$, in contradiction with experiment. The only plausible explanation for the large conductivity (and sign discrepancy) of the $(2 \times 1)$-reconstructed surface seems to be the presence of a surface-state conduction channel.

A second experiment was carried out to determine the surface-state conductance without resorting to temperature-dependent (and model-dependent) spacecharge layer calculations and assumptions about the mobility reduction beneath the $\mathrm{O}_{2}$-passivated surface. In this experiment, we used a 900-Å-thick, fully depleted $p$-type SOI sample to minimize subsurface conduction (Fig. 2 inset). The resistance between the top layer and bottom substrate was only on the order of $1 \mathrm{M} \Omega$ so current does flow through the bottom substrate [21]. However, oxidation of the top layer does not affect the conductance through the substrate beneath the 3800- $\AA$-thick buried oxide. Modeling the SOI as a parallel-plate conductor separated by a nonconducting buried oxide, we thus obtain $G_{2 \times 1}(T)=\sigma_{b}(T) \times d^{\prime}+\Delta \sigma_{s s}(T)$ and $G_{\mathrm{O}_{2}}(T)=$ $\sigma_{b}(T) \times\left[d^{\prime}+t-(1-r) \lambda_{b}(T)\right]$, giving

$\Delta \sigma_{s s}(T)=G_{2 \times 1}(T)-G_{\mathrm{O}_{2}}(T) \frac{d^{\prime}}{d^{\prime}+t-(1-r) \lambda_{b}(T)}$,

where $t$ and $d^{\prime}$ are the thickness of the Si top layer and the supporting substrate, respectively, and $r$ is the Fuchs' specularity parameter of the $\mathrm{O}_{2}$-passivated surface. Note that $\lambda(T)<t \ll d^{\prime}$ for $T>100 \mathrm{~K}$. The experimental surface-state conductance, $\Delta \sigma_{s s}(T)$, is obtained from Eq. (6) and shown in Fig. 4 using $r=0$ (diffuse scattering). In reality, $r$ is a model-dependent parameter that varies with temperature, which also makes $\Delta \sigma_{s s}(T)$ somewhat model dependent. However, its most salient feature, i.e., the sharp increase below $200 \mathrm{~K}$ is model independent because $d^{\prime} \gg t, \lambda(T)$.

The experiment clearly shows that the surface-state conduction increases rapidly below $\sim 200 \mathrm{~K}$. We believe that $\Delta \sigma_{s s}(T)$ decreases with increasing temperature as the buckled dimers on $\mathrm{Si}(100)$ gradually lose their long-range $c(4 \times 2)$ ordering. The order-disorder transition is almost complete near $T \approx 200 \mathrm{~K}$ [22], which explains why $\Delta \sigma_{s s}(T)$ reaches a minimum (maximum surface scattering). To illustrate this point we also show the intensity of the $(3 / 2,3 / 4)$ spot in LEED as a function of $T / T_{c}$ as reported in Ref. [22], which reflects the order parameter of the $c(4 \times 2)$ reconstruction. Figure 4 strongly suggests a link between surface-state conductance and structural (dis)order on the surface. Our own LEED patterns also showed a clear $c(4 \times 2)$ superstructure at the lowest measurement temperature $(120 \mathrm{~K})$. Above 


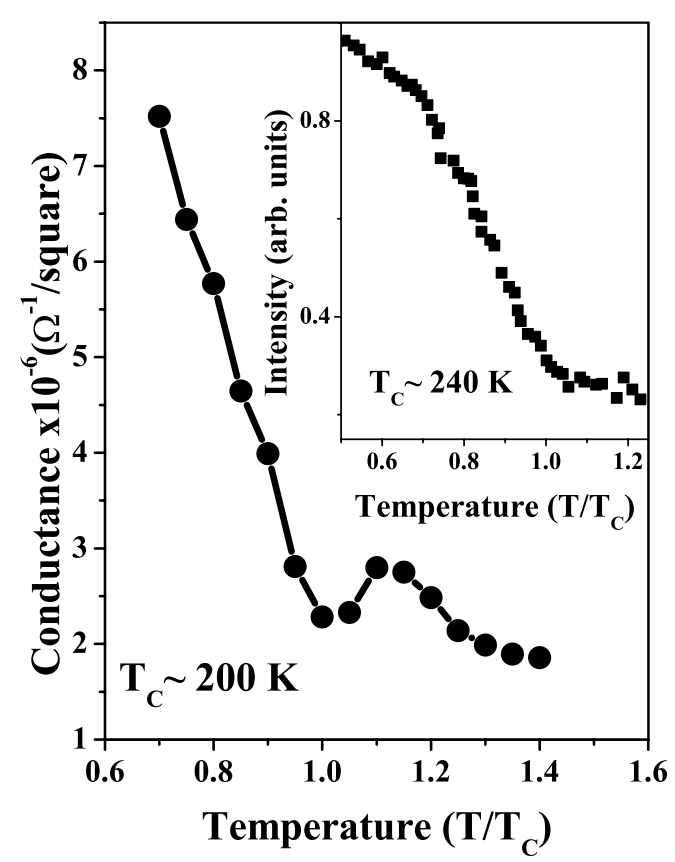

FIG. 4. Experimental determination of the surface-state conductance of (100) SOI, using Eq. (6) with $r=0$. The intensity of the $(3 / 2,3 / 4)$ LEED spot of the $c(4 \times 2)$ superstructure is taken from Ref. [22].

approximately $200 \mathrm{~K}$, fractional order spots were usually too weak to be observed by eye, which is qualitatively consistent with previous observations. There are other surprises. First of all, the surface-state conductivity is sufficiently large to offset the conductivity reduction in the space charge layer beneath the $2 \times 1$ surface. Its magnitude of $\sim 10^{-6} \Omega^{-1} / \square$ is comparable to the RT results by Hasegawa for $\operatorname{Si}(111)-(7 \times 7)$ (which is probably a 2D correlated metal [23]) using scanning tunneling microscopy point contacts [24]. Second, the temperature dependence of the surface-state conductivity is indicative of metallic behavior whereas the $2 \times 1$ and $c(4 \times 2)$ reconstructions have a $\sim 0.5 \mathrm{eV}$ band gap [2]. Electronic transport in this 2D electron system likely involves excitations between the surface-state bands and possibly the type- $C$ gap state (Fig. 1) but evidently, the dominant factor determining the temperature coefficient between 120 and $300 \mathrm{~K}$ is the order parameter of the $c(4 \times 2)$ structure.

In summary, the conductivity of the $\operatorname{Si}(100)-(2 \times 1)$ surface has been measured as a function of temperature on ultrathin SOI and on bulk Si. The surface-state conductance on SOI and bulk Si shows metallic temperature dependence between 120 and $300 \mathrm{~K}$. Measurements on SOI furthermore show a clear signature of the $c(4 \times$ $2) \rightarrow 2 \times 1$ order-disorder transition. The decrease of the surface-state conductance up to $200 \mathrm{~K}$ is attributed to the gradual demise of long-range $c(4 \times 2)$ order on the surface.

We thank D. M. Zehner, J. J. Jellison, O. W. Holland, and P.H. Fleming for their support and help. This work was primarily funded by the NSF under Contract No. DMR 9705246 and in part by the Laboratory Directed Research and Development Program of Oak Ridge National Laboratory, managed by UT-Battelle, LLC, for the U.S. Department of Energy under Contract No. DE-AC05-00OR22725.

[1] J. M. Ziman, Electrons and Phonons (Oxford University Press, Oxford, 1979) (reprinted from 1st ed.).

[2] C. B. Duke, Chem. Rev. 96, 1237 (1996), and references therein.

[3] H. Shigekawa et al., Phys. Rev. B 55, 15448 (1997).

[4] R. J. Hamers and U. K. Kohler, J. Vac. Sci. Technol. A 7, 2854 (1989).

[5] See, e.g., D. Schumacher, Surface Scattering Experiments with Conduction Electrons, Springer Tracts in Modern Physics Vol. 128 (Springer, Berlin, 1993).

[6] B. N. J. Persson, Phys. Rev. B 44, 3277 (1991); J. Chem. Phys. 98, 1659 (1993).

[7] M. Jałochowski and E. Bauer, Phys. Rev. B 37, 8622 (1988).

[8] S. Hasegawa and S. Ino, Phys. Rev. Lett. 68, 1192 (1992).

[9] K. R. Kimberlin and M.C. Tringides, J. Vac. Sci. Technol. A 13, 462 (1995).

[10] S. Heike et al., Phys. Rev. Lett. 81, 890 (1998).

[11] M. Henzler et al., Surf. Sci. 438, 178 (1999).

[12] K. C. Lin et al., Appl. Phys. Lett. 72, 2313 (1998).

[13] H. Ikegami et al., Jpn. J. Appl. Phys. 35, 1593 (1996).

[14] G. Hollinger and F. J. Himpsel, J. Vac. Sci. Technol. A 1, 640 (1983).

[15] C. L. Petersen, F. Grey, and M. Aono, Surf. Sci. 377-379, 676 (1997).

[16] H. Wormeester, E. G. Keim, and A. van Silfhout, Surf. Sci. 271, 340 (1992).

[17] R.F. Greene, Crit. Rev. Solid State Mater. Sci. 4, 477 (1974).

[18] R. H. Kingston and S. F. Neustadter, J. Appl. Phys. 26, 718 (1955).

[19] Y. Goldstein et al., J. Appl. Phys. 32, 2540 (1961).

[20] E. H. Sondheimer, Adv. Phys. 1, 1 (1952).

[21] From ellipsometry measurements the refractive index of the buried oxide layer was 1.612, which compares to 1.46 for pure stoichiometric silica. The substoichiometric oxide in SOI results in poor electrical insulation between top and bottom Si.

[22] M. Kubota and Y. Murata, Phys. Rev. B 49, 4810 (1994); the definition of $T_{c}$ is somewhat arbitrary. It ranges $\sim 200$ to $\sim 250 \mathrm{~K}$ depending on the defect density; see also K. Inoue et al., Phys. Rev. B 49, 14774 (1994).

[23] R. Losio et al., Phys. Rev. B 61, 10845 (2000).

[24] Y. Hasegawa et al., Surf. Sci. 357-358, 32 (1996). 